\title{
THE GEOMETRY OF BANACH SPACES. SMOOTHNESS $\left({ }^{1,2}\right)$
}

BY

\author{
DENNIS F. CUDIA(3)
}

Introduction. This paper contains the first unified treatment of the dual theory of differentiability of the norm functional in a real normed linear space. With this, the work of Šmulian $[2 ; 3]$ is extended and it is shown how uniform convexity is to be modified so as to obtain geometric properties dual to the various types of differentiability of the norm thus answering a question implicit in the work of Lovaglia [1] and Anderson [1]. The resulting dual theory of differentiability is then used to obtain a connection between the differentiability of certain infinite dimensional manifolds imbedded in an infinite dimensional real normed linear space and the continuity properties of the Gaussian spherical image map generalized to such infinite dimensional spaces. In this way a problem proposed by Klee [2, p. 35] is solved.

The principal tools employed are (a) Mazur's characterization [1] of a supporting hyperplane of the unit ball as an inverse image of the derivative of the norm functional; (b) a modification of an integral calculus technique given in Krasnosel'skiī and Rutickī [1, p. 187]; and (c) James' criterion for the reflexivity of a Banach space, viz., that every continuous linear functional attains its supremum on the unit ball. James [1] has given a proof of this criterion for separable Banach spaces and in James [2] has removed the condition that the Banach space be separable.

The paper is divided into five sections. $\$ 1$ contains the localization and directionalization of uniform convexity of Clarkson [1] and of full $k$-convexity of Fan and Glicksberg [1]. §2, while containing interesting facts in its own right, is motivational in nature for $\$ 3 . \S 3$ contains in the dual theory of differentiability of the norm one of the two main conclusions of the paper. The other main conclusion contained in $\S 4$ is the analysis of the differentiability of norm functionals using the generalized Gaussian spherical image map. The last section, $§ 5$, examines and compares the present results in the context of the geometry of Banach spaces as created and perfected by other workers in the field.

\footnotetext{
Received by the editors July 2, 1962.

(1) Research supported by contracts NSF G 12171 and NSF G 19869.

(2) Part of $\$ 4$ was revised in proof July 9, 1963.

(3) This paper is based upon a dissertation submitted in partial fulfillment of the requirements for the Ph. D. degree at the University of Illinois (Urbana).
} 
1. Definitions and notation. The definitions and notation used throughout the paper are collected in this section for easy reference. A good basic reference for the terminology and problems discussed in this paper is the tract Day [3], especially $\$ 2$ of Chapter VII. An historical survey of the geometry of Banach spaces with a more complete bibliography is given in Cudia [1].

All of what follows takes place in a real normed linear space denoted by $X$ or in the dual or conjugate space $X^{*}$ of continuous linear functions on $X$.

1.1. Definition. A convex body in $X$ is a bounded, closed, convex set which has a nonempty interior, and a symmetric convex body is a convex body symmetric about the identity of addition $\theta$ of the linear space.

The obvious symmetric convex body $U=\{x:\|x\| \leqq 1, x \in X\}$ is called the unit ball of $X$ and its boundary $S=\{x:\|x\|=1, x \in X\}$ is the unit sphere of $X$.

In the dual space $X^{*}, U^{\prime}=\left\{f:\|f\| \leqq 1, f \in X^{*}\right\}$ is the unit ball of $X^{*}$ and $S^{\prime}=\left\{f:\|f\|=1, f \in X^{*}\right\}$ is the unit sphere of $X^{*}$. The conjugate norm in $X^{*}$ will be denoted by $\|\cdot\|^{*}$ when it is to be emphasized that the domain of this functional is $X^{*}$, otherwise the asterisk will be omitted. The unit ball and unit sphere of $X^{* *}$ are defined analogously as above and are denoted by $U^{\prime \prime}$ and $S^{\prime \prime}$, respectively. The canonical imbedding of $X$ into $X^{* *}$ or of $X^{*}$ into $X^{* * *}$ will be denoted by $Q$ and $Q_{1}$, respectively.

1.2. Definition. A convex hypersurface is an open connected subset of the boundary of a convex body with the relativized topology on the boundary of the convex body.

The boundary $S$ of $U$ is a convex hypersurface in $X$.

The localization and directionalization of uniform convexity is the subject of the next few definitions.

1.3. Definition. Let $M$ and $N$ be nonempty subsets of $X^{*} . S$ is said to be weakly uniformly rotund at $f$ in the direction $g$ iff for any $\varepsilon>0$ there is a $\delta(\varepsilon, f, g)>0$ such that with $x$ and $y$ in $S,|g(x-y)| \geqq \varepsilon$ implies $\|f\|-1 / 2|f(x+y)| \geqq \delta(\varepsilon, f, g)$. $S$ is said to be weakly uniformly rotund at $M$ in the directions $N$ iff $S$ is weakly uniformly rotund at $f$ in the direction $g$ for any $f$ in $M$ and $g$ in $N . S$ is said to be weakly uniformly rotund uniformly at $M$ in the directions $N$ iff for any $\varepsilon>0$ and $g \in N$, there is a $\delta(\varepsilon, g)>0$ such that, with $x$ and $y$ in $S,|g(x-y)| \geqq \varepsilon$ implies $\|f\|-1 / 2|f(x+y)| \geqq \delta(\varepsilon, g)$ for every $f \in M . S$ is said to be weakly uniformly rotund at $M$ uniformly in the directions $N$ iff for any $\varepsilon>0$ and $f \in M$ there is $\delta(\varepsilon, f)>0$ such that with $x$ and $y$ in $S$, if there is $g \in N$ such that $|g(x-y)| \geqq \varepsilon$ then $\|f\|-1 / 2|f(x+y)|$ $\geqq \delta(\varepsilon, f)$. $S$ is said to be weakly uniformly rotund uniformly at $M$ uniformly in the directions $N$ iff for any $\varepsilon>0$ there is $\delta(\varepsilon)>0$ such that with $x$ and $y$ in $S$, if there is $g \in N$ such that $|g(x-y)| \geqq \varepsilon$ then $\|f\|-1 / 2|f(x+y)| \geqq \delta(\varepsilon)$ for every $f \in M$.

1.4. Definition. If $f$ is a nonzero element in $X^{*}$, then $S$ is said to be weakly uniformly rotund at $f$ in each direction provided $S$ is weakly uniformly rotund 
at $\{f\}$ in the directions $X^{*}$. If $g$ is in $X^{*}$, then $S$ is said to be weakly uniformly rotund in the direction $g$ provided $S$ is weakly uniformly rotund at $X^{*} \sim\{\theta\}$ in the direction $\{g\} . S$ is said to be weakly uniformly rotund in each direction (WURD) iff $S$ is weakly uniformly rotund at $X^{*} \sim\{\theta\}$ in the directions $X^{*}$.

The next definition directionalizes uniform convexity.

1.5. Definition. Let $N$ be a nonempty subset of $X^{*} . S$ is said to be uniformly rotund in the directions $N$ iff $S$ is weakly uniformly rotund uniformly at $S^{\prime}$ in the directions $N$. $S$ is said to be uniformly rotund in each direction (URD) iff $S$ is uniformly rotund in the directions $X^{*}$.

The next definition localizes uniform convexity.

1.6. Definition. Let $M$ be a nonempty subset of $X^{*} \sim\{\theta\}$. Then $S$ is said to be weakly uniformly rotund at $M$ iff $S$ is weakly uniformly rotund at $M$ uniformly in the directions $S^{\prime}$. $S$ is said to be weakly uniformly rotund (WUR) iff $S$ is weakly uniformly rotund at $X^{*} \sim\{\theta\}$.

1.7. DefINITION. $S$ is said to be uniformly rotund (UR) iff $S$ is weakly uniformly rotund uniformly at $S^{\prime}$ uniformly in the directions $S^{\prime}$.

1.8. Definition. If the $x$ and $y$ in Definitions $1.3-1.7$ are merely required to be in $U$ and the implication is satisfied then the word convex will be substituted for the word rotund and $U$ will be said to have the new property. Thus, for example, $U$ is said to be weakly uniformly convex at $f$ in the direction $g$ iff for any $\varepsilon>0$ there is $\delta(\varepsilon, f, g)>0$ such that with $x$ and $y$ in $U,|g(x-y)| \geqq \varepsilon$ implies $\|f\|-1 / 2|f(x+y)| \geqq \delta(\varepsilon, f, g)$.

It is clear how the other properties can be similarly altered.

The following two different types of localizations of uniform convexity have been discussed by Lovaglia [1] and by Anderson [1], respectively.

1.9. Definition. $S$ is said to be locally uniformly rotund iff to any $x_{0} \in S$ and $\varepsilon>0$ there corresponds $\delta\left(\varepsilon, x_{0}\right)>0$ such that with $y \in S,\left\|y-x_{0}\right\| \geqq \varepsilon$ implies $1-1 / 2\left\|x_{0}+y\right\| \geqq \delta\left(\varepsilon, x_{0}\right)$. If the implication is true when $y$ is merely required to be in $U$, then $U$ is said to be locally uniformly convex.

1.10. Definition. $S$ is said to be midpoint locally uniformly rotund iff for any $x_{0} \in S$ and $\varepsilon>0$ there is $\delta\left(\varepsilon, x_{0}\right)>0$ such that with $x$ and $y$ in $S,\|x-y\| \geqq \varepsilon$ implies

$$
\left\|x_{0}-\frac{x+y}{2}\right\| \geqq \delta\left(\varepsilon, x_{0}\right) .
$$

If the implication is valid when $x$ and $y$ are merely restricted to lie in $U$, then $U$ is said to be midpoint locally uniformly convex.

The next definition contains the localization and directionalization of the property called 2-rotundity in Day [3, p. 113] which was first isolated and investigated by Smulian [1]. Here, however, following Fan and Glicksberg [1], $k$-rotundity where $k$ is any integer greater than one will be considered. 
1.11. Definition. Let $\left\{x_{n}\right\}$ be a sequence on $S$. $S$ is said to be weakly $k$-rotund in each direction (WkRD) iff when there is a nonzero $f \in X^{*}$ with the property that

$$
\lim _{n_{1}, \ldots, n_{k} \rightarrow \infty}\left|f\left(\frac{1}{k} \sum_{i=1}^{-} x_{n_{i}}\right)\right|=\|f\|,
$$

then for each $g \in X^{*}$

$$
\lim _{m, n \rightarrow \infty}\left|g\left(x_{m}-x_{n}\right)\right|=0 .
$$

$S$ is said to be weakly $k$-rotund $(\mathrm{W} k \mathrm{R})$ iff when there is $f \in X^{*} \sim\{\theta\}$ such that

$$
\lim _{n_{1}, \ldots, n_{k} \rightarrow \infty}\left|\left(f \frac{1}{k} \sum_{i=1}^{k} x_{n_{1}}\right)\right|=\|f\|
$$

then

$$
\lim _{m, n \rightarrow \infty}\left\|x_{m}-x_{n}\right\|=0
$$

$S$ is said to be $k$-rotund in each direction ( $k \mathrm{RD})$ iff

$$
\lim _{n_{1} \ldots, n_{k} \rightarrow \infty}\left\|\frac{1}{k} \sum_{i=1}^{k} x_{n}\right\|=1
$$

implies that for each $g \in X^{*}$

$$
\lim _{m, n \rightarrow \infty}\left|g\left(x_{m}-x_{n}\right)\right|=0
$$

$S$ is said to be $k$-rotund $(k \mathrm{R})$ iff

$$
\lim _{n_{1}, \ldots, n_{k} \rightarrow \infty}\left\|\frac{1}{k} \sum_{i=1}^{k} x_{n_{i}}\right\|=1
$$

implies that

$$
\lim _{m, n \rightarrow \infty}\left\|x_{m}-x_{n}\right\|=0
$$

As in the previous definitions of this section if $\left\{x_{n}\right\}$ is merely required to be in $U$ and the defining implication is still valid, then the word convex is substituted for the word rotund and $U$ is said to be weakly k-convex in each direction, or weakly $k$-convex, or $k$-convex in each direction, or $k$-convex, whichever the case may be. It is clear from the pattern established in Definition 1.11 how $k$-rotundity can be modified to give properties analogous to the properties given in Definitions 1.3-1.6. However, since only the properties already given in Definition:1.11 will be discussed in this paper these further variations of $k$-rotundity will not be listed.

The following is a well-known property of the unit sphere in a Hilbert space. 
1.12. Definition. $S$ will be said to have property (A) provided that when $\left\{x_{n}\right\}$ is a sequence on $S$ which converges weakly to an element $x_{0}$ on $S$, the sequence also converges in the norm topology to $x_{0}$.

In the proofs of some of the equivalences in this paper the terms "forward implication" and "reverse implication" are used. The former refers to the "only if" part of the statement of the equivalence while the latter refers to the "if", part of the statement of the equivalence.

2. Generalized rotundity and smoothness. In this section the duality between smoothness and rotundity will be examined and it will be shown how a notion due to Klee [3] of generalized rotundity of convex sets in Hausdorff linear spaces gives rise to a better understanding of the nature of this duality. Then a slight modification of this notion will be introduced and examined giving in turn more information on duality and the geometry of quotient spaces of the dual space.

Let $E$ be a Hausdorff linear space, $K$ a convex set in $E$, and $F$ a subset of linear functionals (not necessarily continuous) on $E$. According to Klee [3], a point $p$ of $K$ is said to be an $F$-support point of $K$ if there is an $F$-hyperplane $H=\{x: f(x)=r\}$ for a nonzero $f$ in $F$ and a real number $r$ with the property that $p$ is on $H, K$ is not completely contained in $H$, and $K$ lies on one side of $H$,i. e., either $f(k) \leqq r$ for all $k \in K$ or $f(k) \geqq r$ for all $k \in K$. The $F$-support point $p$ is said to be an $F$-rotund point provided all the $F$-hyperplanes supporting $K$ at $p$ intersect $K$ only at $p$ and $K$ is defined to be $F$-rotund if every $F$-support point is an $F$-rotund point. Klee [3] also generalizes smoothness by defining an $F$-support point $p$ of $K$ to be an $F$-smooth point provided all the $F$-hyperplanes which support $K$ at $p$ have the same intersection with the affine extension of $K . K$ is said to be $F$-smooth if every $F$-support point is an $F$ smooth point. If $K$ has an interior point and $F \supseteq E^{*}$ then every $F$-hyperplane supporting $K$ at a point is a closed set so the linear functional determining it is continuous and $F$-rotundity and $F$-smoothness coincide with the usual notions of rotundity and smoothness. When the letter $F$ is omitted it is to be assumed that the usual notions are being considered.

Assuming that $F$ is a linear subspace of linear functionals on $E, F$-rotundity and $F$-smoothness generalize the usual notions in three ways. The set $K$ may have no interior points and $F$ may or may not contain $E^{*}$, and $K$ may have an interior point and $F$ may be a proper subspace of $E^{*}$. The next theorem considers this first type of generalization.

2.1. THEOREM. Let $X$ be a real linear space and let $E$ be $X^{*}$ with its weak* topology. Then (i) $U$ is smooth iff $U^{\prime}$ is $E^{*}$-rotund, and (ii) $U$ is rotund iff $U^{\prime}$ is $E^{*}$-smooth.

The proof, which depends on the observation that $E^{*}$ is the range of the canonical map $Q$ defined on $X$, is easily constructed and so is omitted. 
If $F$ and $G$ are two linear subspaces of possible support functionals and $F \subseteq G$ then $G$-rotundity implies $F$-rotundity and $G$-smoothness implies $F$-smoothness. If in the situation of Theorem 2.1, $F=E^{*}$ and $G=X^{* *}$ then $X^{* *}$-rotundity of $U^{\prime}$ implies $E^{*}$-rotundity of $U^{\prime}$ and $X^{* *}$-smoothness of $U^{\prime}$ implies $E^{*}$-smoothness of $U^{\prime}$. However, there are cases in which the $X^{* *}$-rotundity of $U^{\prime}$ is definitely stronger than its $E^{*}$-rotundity and in which $X^{* *}$-smoothness of $U^{\prime}$ is definitely stronger than $E^{*}$-smoothness of $U^{\prime}$. Later in this section these counter-examples will be presented.

Although this point of view does give a unified treatment of the elementary duality between smoothness and rotundity, it seems to the author that an even clearer picture is obtained by viewing the situation in terms of the last type of generalization of rotundity discussed in the paragraph preceding Theorem 2.1. Thus in the next theorem the convex set has an interior point and $F$ is contained in the set of all continuous linear functionals.

2.2. THEOREM. Let $X$ be a real normed linear space and let $X^{*}$ be topologized by the conjugate norm. Then (i) $S$ is smooth iff $S^{\prime}$ is $Q(X)$-rotund, and (ii) $S$ is rotund iff $S^{\prime}$ is $Q(X)$-smooth.

Proof. Assertion (i) follows from (i) of Theorem 2.1 with the observation that $S^{\prime}$ is $Q(X)$-rotund iff $U^{\prime}$ is $E^{*}$-rotund. Assertion (ii) follows from (ii) of Theorem 2.1 with the observation that $S^{\prime}$ is $Q(X)$-smooth iff $U^{\prime}$ is $E^{*}$-smooth.

In order to see the connection between $Q(X)$-rotundity and $Q(X)$-smoothness and the geometry of quotient spaces of $X^{*}$ the next lemma is necessary. This lemma was used in Day [1] and a proof is unnecessary.

2.3. LEMMA. (i) $S^{\prime}$ is rotund [smooth] iff every two dimensional quotient space of $X$ with a weakly closed zero element has a smooth [rotund] unit sphere. (ii) $S$ is rotund [smooth] iff every two dimensional quotient space of $X^{*}$ with a weak* closed zero element has a smooth [rotund] unit sphere.

2.4. THEOREM. (i) $S^{\prime}$ is $Q(X)$-rotund iff every two dimensional quotient space of $X^{*}$ with a weak ${ }^{*}$ closed zero element has a rotund unit sphere. (ii) $S^{\prime}$ is $Q(X)$ smooth iff every two dimensional quotient space of $X^{*}$ with a weak* closed zero element has a smooth unit sphere.

Proof. The proof is made by using Theorem 2.2 and (ii) of Lemma 2.3.

The counter-examples mentioned earlier can now be examined. In Day [3, p.114] a symmetric convex body in $l_{1}$ was described which was strictly convex but whose two dimensional quotient spaces were not all strictly convex. The polar body in $m$ according to Lemma 2.3 has all of its two dimensional quotient spaces with a weak* closed zero element smooth but the polar body itself cannot be smooth according to Day [2, p. 522]. This polar body in $m$ is therefore, by Theorem 2.4, $Q(X)$-smooth but not smooth. 
In Klee [3, p. 62] it is proved that there is a separable space with a smooth symmetric convex body but not all of whose two dimensional quotient spaces are smooth. According to Lemma 2.3 then, the polar body has the property that all of its two dimensional quotient spaces with weak* closed zero element are strictly convex but the polar body itself is not strictly convex. This polar body in the dual space is then, according to Theorem 2.4, $Q(X)$-rotund without being rotund. These two examples show that there are rotundity and smoothness conditions which are really weaker than the usual notions in a conjugate space. These counterexamples will be met again in $\$ 5$.

In summary then the restriction of possible supporting hyperplanes of $U^{\prime}$ to inverse images of linear functionals in $Q(X)$ does give rise to useful and interesting geometric conditions. It is clear in this interpretation how complete duality between ordinary rotundity and smoothness is obtained when $U$ is weakly compact, i.e., when $Q(X)=X^{* *}$. Nevertheless, in order to obtain necessary and sufficient dual geometric properties even when the normed space has no weakly compact convex bodies it will be necessary to restrict the set of possible supporting continuous linear functionals in the spirit of Theorem 2.2. The task of localizing various geometric properties demands this point of view and the lack of recognition of this fact has restricted the usefulness of the dual theory of geometric properties by giving only necessary but not sufficient (or vice versa) conditions for one geometric property in one space and its (partial) dual in the other, or by requiring in the hypotheses of the implications that $U$ be weakly compact. In what follows however it will be easily seen how the previously known results are duplicated when the unit ball is weakly compact.

3. Analytical study of smoothness. This section is concerned with the differentiability properties of the norm in a real normed linear space and with extending and completing the theory of geometric properties dual to various types of differentiability of the norm. The section closes with an analysis of the connection between the present work and that of Smulian $[2 ; 3]$.

3.1. Definition. Let $M$ and $N$ be arbitrary nonvacuous subsets of $X$, and consider the following limit

$$
\lim _{\alpha \rightarrow 0} \frac{\|x+\alpha y\|-\|x\|}{\alpha} .
$$

$\|\cdot\|$ is differentiable at $x$ with respect to $y$ iff the limit $\left(^{*}\right)$ exists. $\|\cdot\|$ is differentiable in $M$ with respect to $N$ iff $\|\cdot\|$ is differentiable at $x$ with respect to $y$ for each $x$ in $M$ and $y$ in $N .\|\cdot\|$ is differentiable [uniformly] in $M$ [uniformly] with respect to $N$ iff $\|\cdot\|$ is differentiable in $M$ with respect to $N$ and (*) is approached [uniformly] as $x$ varies in $M$ [uniformly] as $y$ varies in $N$.

3.2. Definition. $\|\cdot\|$ is Gateaux differentiable at $x$ iff $\|\cdot\|$ is differentiable in $\{x\}$ with respect to $S .\|\cdot\|$ is Gateaux differentiable in the direction $y$ iff $\|\cdot\|$ 
is differentiable in $S$ with respect to $\{y\} .\|\cdot\|$ is Gateaux differentiable iff $\|\cdot\|$ is differentiable in $S$ with respect to $S .\|\cdot\|$ is uniformly Gateaux differentiable in the direction $y$ iff $\|\cdot\|$ is differentiable uniformly in $S$ with respect to $\{y\} .\|\cdot\|$ is uniformly Gateaux differentiable iff $\|\cdot\|$ is differentiable uniformly in $S$ with respect to $S$.

3.3. Definition. $\|\cdot\|$ is Frechet differentiable at $x$ iff $\|\cdot\|$ is differentiable in $\{x\}$ uniformly with respect to $S .\|\cdot\|$ is Frechet differentiable iff $\|\cdot\|$ is differentiable in $S$ uniformly with respect to $S .\|\cdot\|$ is uniformly Frechet differentiable iff $\|\cdot\|$ is differentiable uniformly in $S$ uniformly with respect to $S$.

3.4. Definition (Busemann [1, p. 90]). A point $x$ of $X$ is said to be a conical point of $\|\cdot\|$ iff $\|\cdot\|$ is differentiable at $x$ only with respect to the subset $\{r x: r$ real $\}$.

It is easily seen that the origin is a conical point of the norm in any normed linear space and Bonhenblust and Karlin [1] have shown that the identity of multiplication on the surface of the unit ball of a Banach algebra is always a conical point of the norm.

The function $G^{+}(x, y)$ defined by

$$
G^{+}(x, y)=\lim _{\alpha \rightarrow 0^{+}} \frac{\|x+\alpha y\|-\|x\|}{\alpha}
$$

will play a central role in what follows because it always exists and coincides with the limit $\left({ }^{*}\right)$ of Definition 3.1 when the latter exists. The fact of the existence of $G^{+}(x, y)$ for any $x$ and $y$ follows from the observation first made by Ascoli [1, pp. 53-56] in the separable normed space case and by Mazur [1, pp. 75-78] for an arbitrary normed space, that the function $f(\alpha)$ defined for nonzero real $\alpha$ by

$$
f(\alpha)=\frac{\|x+\alpha y\|-\|x\|}{\alpha}
$$

is monotone nondecreasing and is restricted to lie in the closed interval $[-\|y\|,\|y\|]$. Defining $G^{-}(x, y) \equiv-G^{+}(x,-y)$, it is seen that the limit $\left(^{*}\right)$ exists iff $G^{-}(x, y)=G^{+}(x, y)$ and this fact will sometimes be expressed by setting $G(x, y)$ equal to the common value; thus

$$
G(x, y) \equiv \lim _{\alpha \rightarrow 0} \frac{\|x+\alpha y\|-\|x\|}{\alpha} .
$$

The next proposition forms the basis for all the duality results of this section.

3.5. Proposition. Let fand $g$ be in $X^{*}$. (i) If for each $\varepsilon>0$ there is $\eta(\varepsilon, f, g)>0$ such that $0<\alpha \leqq \eta(\varepsilon, f, g)$ implies $\|f+\alpha g\|+\|f-\alpha g\| \leqq 2\|f\|+\alpha \varepsilon$, then setting $\delta(\varepsilon, f, g)=\varepsilon / 4 \eta(\varepsilon / 2, f, g)$, the following implication is valid: with $x$ and $y$ in $S$,

$$
\|f\|-\left|f\left(\frac{x+y}{2}\right)\right|<\delta(\varepsilon, f, g)
$$

implies $|g(x-y)|<\varepsilon$. 
(ii) If for each $\varepsilon>0$ there is $\delta(\varepsilon, f, g)>0$ such that with $x$ and $y$ in $S$

$$
\|f\|-\left|f\left(\frac{x+y}{2}\right)\right|<\delta(\varepsilon, f, g)
$$

implies $|g(x-y)|<\varepsilon$, then setting

$$
\eta(\varepsilon, f, g)=\left\{\begin{array}{cc}
\frac{\delta(\varepsilon, f, g)}{\|g\|}, & g \neq \theta \\
1, & g=\theta
\end{array}\right.
$$

the following implication is valid: $0<\alpha \leqq \eta(\varepsilon, f, g)$ implies

$$
\|f+\alpha g\|+\|f-\alpha g\| \leqq 2\|f\|+\alpha \varepsilon \text {. }
$$

Proof. To prove (i) set $\delta(\varepsilon, f, g)=(\varepsilon / 4) \eta(\varepsilon / 2, f, g)$. Then for any $x$ and $y$ on $S$ and $0<\alpha \leqq \eta(\varepsilon / 2, f, g)$,

Hence

$$
|f(x)+f(y)|+\alpha|g(x)-g(y)| \leqq 2\|f\|+\alpha \varepsilon / 2 .
$$

$$
|g(x)-g(y)| \leqq \frac{\varepsilon}{2}+\frac{2\left[\|f\|-\left|f\left(\frac{x+y}{2}\right)\right|\right]}{\eta(\varepsilon / 2, f, g)}<\varepsilon
$$

if

$$
\|f\|-\left|f\left(\frac{x+y}{2}\right)\right|<\delta(\varepsilon, f, g)
$$

To prove (ii) observe first that if $g=\theta$, the implication is obvious. Hence suppose $g \neq \theta$, in which case $\eta(\varepsilon, f, g)=\|g\|^{-1} \delta(\varepsilon, f, g)$. Then for any $x$ and $y$ in $S$, either

$$
\left|f\left(\frac{x+y}{2}\right)\right|-\|f\| \leqq-\|g\| \eta(\varepsilon, f, g)
$$

or

$$
\left|f\left(\frac{x+y}{2}\right)\right|-\|f\|>-\|g\| \eta(\varepsilon, f, g) .
$$

In the first case, for $0<\alpha \leqq \eta(\varepsilon, f, g)$,

$$
\begin{aligned}
\alpha^{-1}\left\{\left|f\left(\frac{x+y}{2}\right)\right|\right. & -\|f\|\}+\frac{1}{2}|g(x)-g(y)| \\
& \leqq-\alpha^{-1}\|g\| \eta(\varepsilon, f, g)+\frac{1}{2}|g(x)-g(y)| \leqq-\|g\|+\|g\|<\varepsilon / 2 .
\end{aligned}
$$

In the second case,

$$
\|f\|-\left|f\left(\frac{x+y}{2}\right)\right|<\delta(\varepsilon, f, g)
$$


so that for $0<\alpha \leqq \eta(\varepsilon, f, g)$,

$$
\alpha^{-1}\left\{\left|f\left(\frac{x+y}{2}\right)\right|-\|f\|\right\}+\frac{1}{2}|g(x)-g(y)| \leqq \frac{1}{2}|g(x)-g(y)|<\varepsilon / 2 .
$$

Thus if $0<\alpha \leqq \eta(\varepsilon, f, g)$,

$$
f(x)+f(y)+|\alpha g(x)-\alpha g(y)|<2\|f\|+\alpha \varepsilon
$$

for any $x$ and $y$ on $S$. Hence for $0<\alpha \leqq \eta(\varepsilon, f, g)$,

$$
\|f+\alpha g\|+\|f-\alpha g\| \leqq 2\|f\|+\alpha \varepsilon .
$$

3.6. Corollary. Let $x$ and $y$ be elements of $X$.

(i) If for $\varepsilon>0$ there is $\eta(\varepsilon, x, y)>0$ such that $0<\alpha \leqq \eta(\varepsilon, x, y)$ implies $\|x+\alpha y\|+\|x-\alpha y\| \leqq 2\|x\|+\alpha \varepsilon$, then setting $\delta(\varepsilon, x, y) \mid=(\varepsilon / 4) \eta(\varepsilon / 2, x, y)$ the following implication is valid: with $f$ and $g$ in $S^{\prime}$,

implies $|Q y(f-g)|<\varepsilon$.

$$
\|x\|-\left|Q x\left(\frac{f+g}{2}\right)\right|<\delta(\varepsilon, x, y)
$$

(ii) If for each $\varepsilon>0$ there is $\delta(\varepsilon, x, y)>0$ such that with $f$ and $g$ in $S^{\prime}$,

$$
\|x\|-\left|Q x\left(\frac{f+g}{2}\right)\right|<\delta(\varepsilon, x, y)
$$

implies $|Q y(f-g)|<\varepsilon$, then setting

$$
\eta(\varepsilon, x, y)=\left\{\begin{array}{cc}
\frac{\delta(\varepsilon, x, y)}{\|y\|}, & y \neq \theta, \\
1, & y=\theta,
\end{array}\right.
$$

the following implication is valid: $0<\alpha \leqq \eta(\varepsilon, x, y)$ implies

$$
\|x+\alpha y\|+\|x-\alpha y\| \leqq 2\|x\|+\alpha \varepsilon .
$$

3.7. THEOREM. Let $M$ and $N$ be nonvacuous subsets of $X^{*}$. Then $\|\cdot\|^{*}$ is differentiable [uniformly] in $M$ with respect to $N$ iff $S$ is weakly uniformly rotund [uniformly] at $M$ in the directions $N$.

Proof. Because of the monotone nondecreasing nature of the function

$$
f(\alpha)=\frac{\|f+\alpha g\|-\|f\|}{\alpha}
$$

defined and continuous for nonzero $\alpha$, the differentiability condition is equivalent to the following condition: given any $\varepsilon>0$ there is $\eta(\varepsilon, f, g)>0[\eta(\varepsilon, g)>0]$ such that $0<\alpha \leqq \eta(\varepsilon, f, g)[0<\alpha \leqq \eta(\varepsilon, g)]$ implies 


$$
\|f+\alpha g\|+\|f-\alpha g\| \leqq 2\|f\|+\alpha \varepsilon
$$

[for all $f$ in $M$ ]. By Proposition 3.5 the differentiability condition is equivalent to the requirement that to each triple [pair] $\varepsilon>0, f$ in $M$, and $g$ in $N[\varepsilon>0$, and $g$ in $N]$ there should correspond $\delta(\varepsilon, f, g)>0[\delta(\varepsilon, g)>0]$ such that with $x$ and $y$ in $S$

$$
\|f\|-\left|f\left(\frac{x+y}{2}\right)\right|<\delta(\varepsilon, f, g), \quad[\delta(\varepsilon, g)]
$$

[for some $f$ in $M$ ] implies $|g(x-y)|<\varepsilon$. Taking the contrapositive it is seen that the differentiability condition is equivalent to the requirement that to any triple [pair] $\varepsilon>0, f$ in $M$, and $g$ in $N[\varepsilon>0$, and $g$ in $N]$ there is a $\delta(\varepsilon, f, g)>0[\delta(\varepsilon, g)>0]$ such that with $x$ and $y$ in $S,|g(x-y)| \geqq \varepsilon$ implies

$$
\|f\|-\left|f\left(\frac{x+y}{2}\right)\right| \geqq \delta(\varepsilon, f, g), \quad[\delta(\varepsilon, g)]
$$

[for all $f$ in $M]$.

3.8. Corollary. Let $M$ and $N$ be nonvacuous subsets of $X$. Then $\|\cdot\|$ is differentiable [uniformly] in $M$ with respect to $N$ iff $S^{\prime}$ is weakly uniformly rotund [uniformly] at $Q(M)$ in the directions $Q(N)$.

3.9. Corollary. (i) Let $x$ and $y$ be in $X$ with $x$ a point on $S$. Theni $\|\cdot\|$ is differentiable at $x$ with respect to $y$ iff $S^{\prime}$ is weakly uniformly rotund at $Q x$ in the direction $Q y$. (ii) Let $f$ and $g$ be in $X^{*}$ with $f$ on $S^{\prime}$. Then $\|\cdot\|^{*}$ is differentiable at $f$ with respect to $g$ iff $S$ is weakly uniformly rotund at $f$ in the direction $g$.

3.10. CoROllary. (i) Let $x$ be a point on $S$. Then $\|\cdot\|$ is Gateaux differentiable at $x$ iff $S^{\prime}$ is weakly uniformly rotund at $Q x$ in the directions $Q(S)$. (ii) Let $f$ be a point on $S^{\prime}$. Then $\|\cdot\|^{*}$ is Gateaux differentiable at $f$ iff $S$ is weakly uniformly rotund at $f$ in each direction.

3.11. COROLlarY. (i) Let $y$ be any element other than the neutral element in $X$. Then $\|\cdot\|$ is Gateaux differentiable in the direction $y$ iff $S^{\prime}$ is weakly uniformly rotund at $Q(S)$ in the direction $Q y$. (ii) Let $g$ be any element other than the neutral element in $X^{*}$. Then $\|\cdot\|^{*}$ is Gateaux differentiable in the direction $g$ iff $S$ is weakly uniformly rotund in the direction $g$.

In the next corollary note how the weak compactness of the unit ball is needed to insure complete duality between Gateaux differentiability and weak uniform rotundity in each direction.

3.12. COROLlary. (i) $\|\cdot\|$ is Gateaux differentiable iff $S^{\prime}$ is weakly uniformly rotund at $Q(S)$ in the directions $Q(S)$. (ii) $\|\cdot\|^{*}$ is Gateaux differentiable iff $S$ is weakly uniformly rotund in each direction. 
Uniform Gateaux differentiability will be considered in the remaining corollaries to Theorem 3.7.

3.13. CoRollary. (i) Let $y$ be any element other than the neutral element in $X$. Then $\|\cdot\|$ is uniformly Gateaux differentiable in the direction $y$ iff $S^{\prime}$ is uniformly rotund in the direction $Q y$. (ii) Let $g$ be any element other than the neutral element in $X^{*}$. Then $\left\|^{*}\right\|^{*}$ is uniformly Gateaux differentiable in the direction $g$ iff $S$ is uniformly rotund in the direction $g$.

In the next corollary note how weak compactness of $U$ insures complete duality between uniform Gateaux differentiability and uniform rotundity in each direction.

3.14. CoRollary. (i) $\|\cdot\|$ is uniformly Gateaux differentiable iff $S^{\prime}$ is uniformly rotund in the directions $Q(S)$. (ii) $\|\cdot\|^{*}$ is uniformly Gateaux differentiable iff $S$ is uniformly rotund in each direction.

3.15. THEOREM. Let $M$ and $N$ be nonvacuous subsets of $X^{*}$. Then $\|\cdot\|^{*}$ is differentiable [uniformly] in $M$ uniformly with respect to $N$ iff $S$ is weakly uniformly rotund [uniformly] at $M$ uniformly in the directions $N$.

Proof. To prove the forward implication observe that the differentiability condition gives an $\eta(\varepsilon, f)[\eta(\varepsilon)]$ which when used with Proposition 3.5 gives a $\delta(\varepsilon, f)[\delta(\varepsilon)]$ such that with $x$ and $y$ on $S$,

implies that

$$
\exists g \in N \ni|g(x-y)| \geqq \varepsilon
$$

$$
\|f\|-\left|f\left(\frac{x+y}{2}\right)\right| \geqq \delta(\varepsilon, f), \quad[\delta(\varepsilon)]
$$

[for all $f$ in $M$ ].

To prove the reverse implication first observe that the rotundity condition gives the existence for each pair $\varepsilon>0$, and $f$ in $M$, of a $\delta(\varepsilon, f)[\delta(\varepsilon)]$ such that with $x$ and $y$ in $S,[\exists f$ in $M$ such that $]$

$$
\|f\|-\left|f\left(\frac{x+y}{2}\right)\right|<\delta(\varepsilon, f), \quad[\delta(\varepsilon)]
$$

implies $|g(x-y)|<\varepsilon$ for all $g$ in $N$. This implies that $N$ is a bounded set. Ignoring the case when $N=\{\theta\}$, set $B=\sup \{\|g\|: g \in N\}$ so that $0<B<\infty$. Substituting $B$ for $\|g\|$ in the definition of $\eta(\varepsilon, f, g)$ in (ii) of Proposition 3.5 gives an $\bar{\eta}(\varepsilon, f)$ $[\bar{\eta}(\varepsilon)]$ such that $0<\alpha \leqq \bar{\eta}(\varepsilon, f)[0<\alpha \leqq \bar{\eta}(\varepsilon)]$ implies $\|f+\alpha g\|+\|f-\alpha g\|$ $\leqq 2\|f\|+\alpha \varepsilon$ for all $g$ in $N$ [and, simultaneously, for all $f$ in $M$ ].

3.16. Corollary. Let $M$ and $N$ be nonvacuous subsets of $X$. Then $\|\cdot\|$ is differentiable [uniformly] in $M$ uniformly with respect to $N$ iff $S^{\prime}$ is weakly uniformly rotund [uniformly] at $Q(M)$ uniformly in the directions $Q(N)$. 
3.17. COROLLARY. (i) Let $x$ be a point on $S$. Then $\|\cdot\|$ is Frechet differentiable at the point $x$ iff $S^{\prime}$ is weakly uniformly rotund at $Q x$. (ii) Let $f$ be a point on $S^{\prime}$. Then $\|\cdot\|^{*}$ is Frechet differentiable at $f$ iff $S$ is weakly uniformly rotund at $f$.

In the next corollary note how the weak compactness of $U$ allows complete duality between Frechet differentiability and weak uniform rotundity.

3.18. COROLLARY. (i) $\|\cdot\|$ is Frechet differentiable iff $S^{\prime}$ is weakly uniformly rotund at $Q(S)$. (ii) $\|\cdot\|^{*}$ is Frechet differentiable iff $S$ is weakly uniformly rotund.

Uniform Frechet differentiability is considered in the next corollary. Note that no completeness or compactness condition is needed for complete duality between uniform Frechet differentiability and uniform rotundity.

3.19. COROLLARY. (i) $\|\cdot\|$ is uniformly Frechet differentiable iff $S^{\prime}$ is uniformly rotund. (ii) $\|\cdot\|^{*}$ is uniformly Frechet differentiable iff $S$ is uniformly rotund.

In order to bring out the connection between the theorems and corollaries of this section and the work of Smulian $[2 ; 3]$ on the dual theory of differentiability of the norm, several easily proved equivalences will be stated. The equivalences are between an implication and its contrapositive. The unbracketed sequential property stated in the next theorem is called (D) (or $\left(D^{*}\right)$ in the dual space) in the book of Day [3, p. 112] and in Cudia [1], and it is the property (E.2) in the paper of Fan and Glicksberg [2].

3.20. THEOREM. (i) If $f_{0}$ is in $S^{\prime}$ then $S$ is weakly uniformly rotund at $f_{0}$ [in each direction] iff $\left\|x_{n}\right\|=1$ and $\lim _{n} f_{0}\left(x_{n}\right)=1$ implies that $\left\{x_{n}\right\}$ is a Cauchy sequence in the norm [weak] topology. (ii) If $x_{0}$ is in $S$ then $S^{\prime}$ is weakly uniformly rotund at $Q x_{0}$ [in the directions $\left.Q(X)\right]$ iff $\left\|f_{n}\right\|=1$ and $\lim _{n} f_{n}\left(x_{0}\right)=1$ implies that $\left\{f_{n}\right\}$ is a Cauchy sequence in the norm [weak*] topology.

In [3] ([2]) Šmulian showed by a sequential argument that the sequential conditions of Theorem 3.20 were equivalent to the Frechet [Gateaux] differentiability of $\|\cdot\|^{*}$ and $\|\cdot\|$.

The unbracketed property stated in the next theorem was first recognized by Smulian [3] as a reformulation of uniform convexity. Ruston [1] later proved the same equivalence. The property is called (UD) in Day [3, p. 113].

3.21. THEOREM. (i) $S$ is uniformly rotund [in each direction] iff with $x$ and $y$ in $S$

$$
\begin{aligned}
& f(x)>1-\delta(\varepsilon),[\delta(\varepsilon, g)], \\
& f(y)>1-\delta(\varepsilon),[\delta(\varepsilon, g)],
\end{aligned}
$$

for some $f$ in $S^{\prime}$ implies $\|x-y\|<\varepsilon[|g(x)-g(y)|<\varepsilon]$. (ii) $S^{\prime}$ is uniformly rotund [in the directions $Q(X)]$ iff with $f$ and $g$ in $S^{\prime}$ 


$$
\begin{gathered}
f(x)>1-\delta(\varepsilon),[\delta(\varepsilon, y)], \\
g(x)>1-\delta(\varepsilon),[\delta(\varepsilon, y)],
\end{gathered}
$$

for some $x$ in $S$ implies that $\|f-g\|<\varepsilon[|f(y)-g(y)|<\varepsilon]$.

Again by a sequential argument Smulian in [3] showed that the conditions in Theorem 3.21 were equivalent to the uniform Frechet [Gateaux] differentiability of $\|\cdot\|^{*}$ and $\|\cdot\|$.

4. Topological study of smoothness. The setting for this section is a linear space $X$ with norm $\|\cdot\|$. The classical notion of the spherical image of a surface in three dimensions will be generalized to convex hypersurfaces in $X$, i.e., open connected subsets of boundaries of convex sets with an interior point. If $X$ is an $n$-dimensional Euclidean space and $C$ is a convex hypersurface in $X$ let $v_{x}$ be the point on the unit sphere which is the endpoint of the unit vector at the origin whose translation is an outward unit normal to $C$ at $x$. Since each point $x$ on such a convex hypersurface has a hyperplane of support at $x$, the unit outward normal to this hyperplane at $x$ is well defined and the endpoint of this normal on the unit sphere when translated to the origin is the image of $x$ on the unit sphere or the spherical image of $x$. Note that $v_{x}$ may be many-valued depending on the existence of corners on the convex hypersurface at $x$. For $X$ a finite dimensional Euclidean space it has long been known (cf. Busemann $[1$, p. 6]) that if the convex hypersurface is differentiable then the spherical image map is continuous. Thus with the generalizations of differentiability isolated in $\$ 3$ the topological properties of the spherical image map may be re-examined in the light of these differing assumptions on the differentiability of the Minkowski functional of the convex set whose boundary contains the convex hypersurface. However in a general normed space where no obvious notion of orthogonality is at hand the spherical image of $x$ must consist of elements on $S^{\prime}$. Thus the definition of the generalized notion of spherical image comes to be as follows.

4.1. Definition. Let $C$ be an open connected subset of the boundary of a convex set $K$ with an interior point. For any $x$ in $C$ let $v_{x}$ be the set of elements $f$ in $S^{\prime}$ with the property that there is a positive number $r$ such that $f^{-1}(r)$ is a hyperplane of support of $K$ at $x$. The set $v_{x}$ is defined to be the spherical image of $x$. The spherical image of a subset of $C$ will be defined to be the set theoretic union of the spherical images of the points in the subset.

4.2. Definition. A point to set map $\phi$ from the topological space $X$ to sets in the topological space $Y$ is said to be upper semicontinuous iff for any net $\left\{x_{d}\right\}$ approaching $x$ and $y_{d}$ in $\phi\left(x_{d}\right)$, if the net $\left\{y_{d}\right\}$ approaches $y$, then $y \in \phi(x) . \phi$ is said to be lower semicontinuous iff for any $y$ in $\phi(x)$ and $V(y)$ a neighborhood of $y$ in $Y$, there is a neighborhood $W(x)$ of $x$ in $X$ such that $\phi\left(x^{\prime}\right) \cap V(y) \neq \varnothing$ for all $x^{\prime}$ in $W(x)$. If $X$ and $Y$ are subsets of linear topological spaces with the 
relativized topology and $W$ depends only on $V$, then $\phi$ is said to be uniformly lower semicontinuous.

Note that if $\phi$ is single-valued then upper semicontinuity of $\phi$ means that the graph of $\phi$ is closed and lower semicontinuity means simply that $\phi$ is continuous.

In the remainder of this section the spherical image of the boundary of symmetric convex bodies will be considered. Since the Minkowski functional of such a convex set is an isomorphic norm to the fixed norm $\|\cdot\|$ of the space, the functional will be denoted by $\|\cdot\|_{1}$ and the surface $S_{1}$ will then be given by $S_{1}=\left\{x:\|x\|_{1}=1\right\}$. The polar set to $U_{1}=\left\{x:\|x\|_{1} \leqq 1\right\}$ will be denoted by $U_{1}^{\prime}$ and its Minkowski functional by $\|\cdot\|_{1}$. The boundary of $U_{1}^{\prime}$ will be denoted and defined by $S_{1}^{\prime}=$ $\left\{f:\|f\|_{1}=1, f \in X^{*}\right\}$. The theorems in this section will show that the smoothness of $S$ and $S_{1}$ is reflected very clearly in the continuity properties of the spherical image mapping $v$ defined on $S$ or $S_{1}$. The section closes with the solution of a problem proposed by Klee [2, p. 35].

4.3. TheOREM. Let $v$ be the spherical image map defined on $S$. Then $v$ is always upper semicontinuous from the norm topology relativized to $S$ into $S^{\prime}$ with its relativized weak* topology.

Proof. Suppose that $\left\{x_{d}\right\}$ is a net of points on $S$ approaching $x$ and $f_{d} \in v_{x_{d}}$ with $\left\{f_{d}\right\}$ a net of points of norm one approaching $f$ in the weak* topology relativized to $S^{\prime}$. Since the conjugate norm is a weak* lower semicontinuous functional,

$$
1=\liminf _{d}\left\|f_{d}\right\| \geqq\|f\|
$$

Also

$$
\left|\left\|f_{d}\right\|-f_{d}(x)\right|=\left|f_{d}\left(x_{d}\right)-f_{d}(x)\right| \leqq\left\|x_{d}-x\right\|
$$

Hence

$$
f(x)=\lim _{d} f_{d}(x)=\lim _{d}\left\|f_{d}\right\| \geqq\|f\|
$$

Thus $\|f\|=1$ and $f(x)=\|f\|$ so that $f \in v_{x}$, showing that $v$ defined on $S$ is upper semicontinuous.

4.4. THEOREM. Let $M$ and $N$ be subsets of $S$. Then $\|\cdot\|$ is differentiable at $M$ with respect to $N$ iff $v$ is lower semicontinuous on $M$ from the norm topology relativized to $S$ into $S^{\prime}$ with the relativized $\sigma\left(X^{*}, N\right)$ topology.

Proof. The forward implication will require an inequality which will be used in the proofs of other theorems in this section. If $x$ and $z$ are on $S$ with $f \in v_{x}$ and $g \in v_{z}$, then 


$$
\begin{aligned}
\|x\|-\left|Q x\left(\frac{f+g}{2}\right)\right| & =\|x\|-\left|\frac{\|x\|+g(x)}{2}\right| \\
& =\|x\|-\left|\frac{\|x\|+\|z\|}{2}+\frac{g(x-z)}{2}\right| \\
& \leqq\|x\|-\frac{\|x\|+\|z\|}{2}+\frac{g(z-x)}{2} \\
& \leqq \frac{\|x\|-\|z\|}{2}+\frac{g(z-x)}{2} \\
& \leqq \frac{\|x-z\|}{2}+\frac{\|x-z\|}{2}
\end{aligned}
$$

Hence if $x$ and $z$ are points on $S$ and $f \in v_{x}$ and $g \in v_{z}$, then

$$
\|x\|-\left|Q x\left(\frac{f+g}{2}\right)\right| \leqq\|x-z\| .
$$

To continue with the proof of the forward implication, let $x \in M, h \in v_{x}$, and $V(h)$ be any neighborhood of $h$ in the relativized $\sigma\left(X^{*}, N\right)$ topology. Then there is a finite set $y_{1}, \cdots, y_{n}$ of elements in $N$ and $\varepsilon>0$ such that

$$
\left|f\left(y_{i}\right)-h\left(y_{i}\right)\right|<\varepsilon \quad i=1, \cdots, n
$$

mplies that $f \in V(h)$. By Corollary 3.8 for each $i$, there is a $\delta_{i}\left(\varepsilon, x, y_{i}\right)>0$ such that for $f$ and $g$ in $S^{\prime}$,

$$
\|x\|-\left|Q x\left(\frac{f+g}{2}\right)\right|<\delta_{i}\left(\varepsilon, x, y_{i}\right)
$$

implies $\left|f\left(y_{i}\right)-g\left(y_{i}\right)\right|<\varepsilon$. Now let

$$
\delta\left(x ; y_{1}, \cdots, y_{n}, \varepsilon\right)=\min _{1 \leqq i \leqq n} \delta_{i}\left(\varepsilon, x, y_{i}\right) .
$$

Then for $z$ on $S$ with $\|x-z\|<\delta\left(x ; y_{1}, \cdots, y_{n}, \varepsilon\right)$, by $\left(^{*}\right)$ for every $f$ in $v_{z}$,

$$
\| x_{\|}-\left|Q x\left(\frac{f+h}{2}\right)\right|<\delta\left(x ; y_{1}, \cdots, y_{n}, \varepsilon\right)
$$

so $\left|f\left(y_{i}\right)-h\left(y_{i}\right)\right|<\varepsilon$ for $i=1, \cdots, n$ so that $f \in V(h)$ and therefore $v$ is lower semicontinuous at $x$ in the relevant topologies.

To prove the reverse implication let $x$ be any element in $M$ and let $f$ and $g$ be elements in $v_{x}$. It will be shown that $f(y)=g(y)$ for every $y$ in $N$ and this will suffice to complete the proof of the theorem.

Let $y \neq x$ be any element in $N$ and denote by $Y$ the two dimensional subspace through $x$ and $y$. Then $Y$ with the relativized norm topology is a separable Banach 
space and $U \cap Y$ is a convex set with an interior point in this relativized norm topology. Hence by a theorem of Mazur [1] the points on $S \cap Y$ with a unique tangent are dense in the norm topology of $X$ relativized to $S \cap Y$. Thus for a dense set of points on $S \cap Y$, the restrictions to $Y$ of elements in $S^{\prime}$ which are supporting linear functionals at a fixed point in this dense set are identical.

Now let $V(f ; y, \varepsilon / 2)$ and $V(g ; y, \varepsilon / 2)$ be $\sigma\left(X^{*}, N\right)$ neighborhoods of $f$ and $g$ respectively for a given positive $\varepsilon$. Then because $v$ by assumption is lower semicontinuous at $x$, there are positive numbers $\delta_{1}$ and $\delta_{2}$ such that $\|x-u\|<\delta_{1}$ implies $v_{v} \cap V(f ; y, \varepsilon / 2) \neq \varnothing$ and $\|x-v\|<\delta_{2}$ implies $v_{u} \cap V(g ; y, \varepsilon / 2) \neq \varnothing$. Let $\delta=\min \left\{\delta_{1}, \delta_{2}\right\}$ and select an element $d$ of $S \cap Y$ of the dense set referred to above such that $\|x-d\|<\delta$. Then $v_{d} \cap V(f ; y, \varepsilon / 2) \neq \varnothing$ and $v_{d} \cap V(g ; y, \varepsilon / 2)$ $\neq \varnothing$. Thus there are elements $h_{1}$ and $h_{2}$ in $v_{d}$ such that $\left|h_{1}(y)-f(y)\right|<\varepsilon / 2$ and $\left|h_{2}(y)-g(y)\right|<\varepsilon / 2$. But because of the uniqueness of the tangent at the point $d$ relative to the space $Y, h_{1}(y)=h_{2}(y)$ so that $|f(y)-g(y)|<\varepsilon$. Since $\varepsilon$ was arbitrary and $y$ was an arbitrary element in $N, f(y)=g(y)$ for all $y$ in $N$. Hence since $x$ was an arbitrary element of $M,\|\cdot\|$ is differentiable in $M$ with respect to $N$.

\subsection{COROLLARY. The following are equivalent:}

(i) $\|\cdot\|$ is Gateaux differentiable at a point $x$ of $S$;

(ii) $v$ is single-valued at $x$;

(iii) $v$ defined on $S$ is lower semicontinuous at $x$ from the norm topology on $S$ into the weak* topology on $S^{\prime}$.

The equivalence between (i) and (ii) of the preceding corollary is due to Mazur [1] while an assertion equivalent to (i) implies (iii) was stated but proved in a different way in Smulian $[2$, p. 86].

4.6. TheOREM. Let $M$ and $N$ be subsets of $S$. Then $\|\cdot\|$ is differentiable uniformly in $M$ with respect to $N$ iff $v$ defined on $S$ is uniformly lower semicontinuous in $M$ from the norm topology on $S$ into the $\sigma\left(X^{*}, N\right)$ topology on $S^{\prime}$.

Proof. The first half of the proof of Theorem 4.4 together with the observation that the differentiability condition implies that the $\delta\left(x ; y_{1}, \cdots, y_{n}, \varepsilon\right)$ of that proof is independent of $x$ for $x$ in $M$, gives a proof of the forward implication of the present theorem.

To prove the reverse implication first observe that because of the equivalence stated in Theorem 4.4 the limit

$$
\lim _{\alpha \rightarrow 0} \frac{\|x+\alpha y\|-\|x\|}{\alpha}=G(x, y)
$$

exists for every $x$ in $M$ and $y$ in $N$. Next observe that for any $x$ and $y$ in $X$ and any real $t$, the real-valued function of the real variable $\alpha$ for $0 \leqq \alpha \leqq 1$, 


$$
F(\alpha)=\|x+t \alpha y\|
$$

is absolutely continuous on [0,1]. Hence by standard theorems of real analysis $F(\alpha)$ has a finite derivative almost everywhere on $[0,1], F^{\prime}(\alpha)$ is Lebesgue integrable on $[0,1]$, and

$$
\|x+t y\|-\|x\|=\int_{0}^{1} \frac{d}{d \alpha}\|x+t \alpha y\| d \alpha
$$

for any $x$ and $y$ in $X$ and any real $t$. Therefore

$$
\frac{d}{d \alpha}\|x+t \alpha y\|=\lim _{\beta \rightarrow 0} \frac{\|x+t \alpha y+t \beta y\|-\|x+t \alpha y\|}{\beta}=G(x+t \alpha y, t y)
$$

exists for $\alpha$ in the complement of some set (depending on $x, y$, and $t$ ) of Lebesgue measure zero. Thus for each $x \in M, y \in N$, and real $t$,

$$
\left|\frac{\|x+t y\|-\|x\|}{t}-G(x, y)\right| \leqq \int_{0}^{1}|G(x+t \alpha y, y)-G(x, y)| d \alpha .
$$

Now, if $x$ and $y$ are on $S$ and $0 \leqq \alpha \leqq 1$, then

$$
\begin{aligned}
\left\|x-\frac{x+t \alpha y}{\|x+t \alpha y\|}\right\| & \leqq\|x-(x+t \alpha y)\|+\left\|x+t \alpha y-\frac{x+t \alpha y}{\|x+t \alpha y\|}\right\| \\
& \leqq|t \alpha|+\frac{\|(\|x+t \alpha y\|-1) x\|}{\|x+t \alpha y\|}+\frac{\|(\|x+t \alpha y\|-1) t \alpha y\|}{\|x+t \alpha y\|} \\
& \leqq|t \alpha|+\frac{|\|x+t \alpha y\|-\|x\||}{\|x+t \alpha y\|}+\frac{|t \alpha||\|x+t \alpha y\|-\|x\||}{\|x+t \alpha y\|} \\
& \leqq|t \alpha|+\frac{|t \alpha|+|t \alpha|^{2}}{\|x+t \alpha y\|} \\
& \leqq|t \alpha|+\frac{|t \alpha|+|t \alpha|^{2}}{|1-| t \alpha||} \\
& \leqq|t|+\frac{|t|+|t|^{2}}{|1-| t||}
\end{aligned}
$$

Since for any positive $\beta, G(x, y)=G(\beta x, y)$ for all $y$,

$$
|G(x+t \alpha y, y)-G(x, y)|=\left|G\left(\frac{x+t \alpha y}{\|x+t \alpha y\|}, y\right)-G(x, y)\right| .
$$

Now let $\varepsilon>0$ and $y_{0}$ in $N$ be given. Then since $v$ is uniformly lower semicontinuous in $M$ from the norm topology on $S$ into $S^{\prime}$ with its relativized $\sigma\left(X^{*}, N\right)$ 
topology, there is a $\delta\left(\varepsilon, y_{0}\right)>0$ such that with $x$ and $z$ in $M$ and $f$ in $v_{x}$, $\|x-z\|<\delta\left(\varepsilon, y_{0}\right)$ implies there exists a $g$ in $v_{z}$ such that $\left|f\left(y_{0}\right)-g\left(y_{0}\right)\right|<\varepsilon$. According to inequality (2) there is an $\eta\left(\varepsilon, y_{0}\right)>0$ such that $|t|<\eta\left(\varepsilon, y_{0}\right)$ implies that

$$
\left\|\frac{x+t \alpha y_{0}}{\left\|x+t \alpha y_{0}\right\|}-x\right\|<\delta\left(\varepsilon, y_{0}\right)
$$

for any $x$ in $M$ and all $0 \leqq \alpha \leqq 1$. Now let $t_{0}$ be a fixed real number such that $0<\left|t_{0}\right|<\eta\left(\varepsilon, y_{0}\right)$ and let $x_{0}$ be an arbitrary but then fixed element of $M$. Then except for a set of $\alpha$ of Lebesgue measure zero which depends on $x_{0}, y_{0}$, and $t_{0}$, $G\left(\left(x_{0}+t_{0} \alpha y_{0}\right) /\left\|x_{0}+t_{0} \alpha y_{0}\right\|, y_{0}\right)$ exists. But it is clear that whenever $G\left(\left(x_{0}+t_{0} \alpha y_{0}\right) /\left\|x_{0}+t_{0} \alpha y_{0}\right\|, y_{0}\right)$ exists, any $g_{\alpha} \in v_{\left(x_{0}+t_{0} \alpha y_{0}\right) /\left\|x_{0}+t_{0} \alpha y_{0}\right\|}$ has the property that

$$
g_{\alpha}\left(y_{0}\right)=G\left(\frac{x_{0}+t_{0} \alpha y_{0}}{\left\|x_{0}+t_{0} \alpha y_{0}\right\|}, y_{0}\right)
$$

Hence if $f \in v_{x_{0}}$ then

$$
\begin{aligned}
G\left(x_{0}+t_{0} \alpha y_{0}, y_{0}\right)-G\left(x_{0}, y_{0}\right) & =G\left(\frac{x_{0}+t_{0} \alpha y_{0}}{\left\|x_{0}+t_{0} \alpha y_{0}\right\|}, y_{0}\right)-G\left(x_{0}, y_{0}\right) \\
& =g_{\alpha}\left(y_{0}\right)-f\left(y_{0}\right)
\end{aligned}
$$

for almost all $\alpha$. Hence using the hypothesis of uniform lower semicontinuity we see that the integrand in (1) is less than $\varepsilon$ for almost all $\alpha$. Hence by (1),

$$
\left|\frac{\left\|x_{0}+t_{0} y_{0}\right\|-\left\|x_{0}\right\|}{t_{0}}-G\left(x_{0}, y_{0}\right)\right|<\varepsilon .
$$

Thus for all $t$ such that $|t|<\eta\left(\varepsilon, y_{0}\right)$

$$
\left|\frac{\left\|x_{0}+t y_{0}\right\|-\left\|x_{0}\right\|}{t}-G\left(x_{0}, y_{0}\right)\right|<\varepsilon .
$$

Since $x_{0}$ was an arbitrary element in $M$ and $\eta\left(\varepsilon, y_{0}\right)$ is independent of $x_{0}$, the reverse implication of the theorem is proved.

4.7. Corollary. If $M$ is a subset of $S$, then $\|\cdot\|$ is differentiable uniformly in $M$ with respect to $X$ iff $v$ defined on $S$ is uniformly lower semicontinuous in $M$ from the norm topology on $S$ into the weak* topology on $S^{\prime}$.

The following corollary uniformizes Corollary 4.5 in a certain way. Another uniformization is given in Corollary 4.12.

4.8. Corollary. Let $C$ be a convex hypersurface contained in $S$. Then the following are equivalent: 
(i) $\|\cdot\|$ is [uniformly] Gateaux differentiable in $C$;

(ii) $v$ defined on $C$ is single-valued and [uniformly] continuous from the norm topology on $C$ into the weak* topology on $S^{\prime}$;

(iii) $v$ defined on $C$ is [uniformly] lower semicontinuous in $C$ from the norm topology on $C$ into the weak* topology on $S^{\prime}$.

4.9. THEOREM. Let $M$ and $N$ be subsets of $S$. Then $\|\cdot\|$ is differentiable [uniformly] in $M$ uniformly with respect to $N$ iff $v$ defined on $S$ is [uniformly] lower semicontinuous in $M$ from the norm topology relativized to $S$ into $S^{\prime}$ with the relativized topology generated by the seminorm $p_{N}(f)=\sup _{y \in N}|f(y)|$.

Proof. To prove the forward implication let $x \in M, h \in v_{x}$, and $V(h)$ be a neighborhood of $h$ in the $p_{N}$ topology. Then there is an $\varepsilon>0$ such that $p_{N}(f-h)<\varepsilon$ implies $f \in V(h)$. According to Corollary 3.16 there is a $\delta(x, \varepsilon / 2)>0[\delta(\varepsilon / 2)>0]$ such that with $f$ and $g$ in $S^{\prime}$

$$
\|x\|-\left|Q x\left(\frac{f+g}{2}\right)\right|<\delta(x, \varepsilon / 2), \quad[\delta(\varepsilon / 2)]
$$

implies $|Q y(f-g)|<\varepsilon / 2$ for all $y$ in $N$. Hence by $\left(^{*}\right)$ of the proof of Theorem 4.4, if $\|x-u\|<\delta(x, \varepsilon / 2), \quad[\delta(\varepsilon / 2)]$, then with $f \in v_{u}$,

$$
\|x\|-\left|Q x\left(\frac{f+h}{2}\right)\right|<\delta(x, \varepsilon / 2), \quad[\delta(\varepsilon / 2)]
$$

so that $p_{N}(f-h)<\varepsilon$ and $f \in V(h)$. Thus $v_{u} \cap V(h) \neq \varnothing$ and $v$ is [uniformly] lower semicontinuous in the relevant topologies.

The proof of the reverse implication proceeds exactly as the proof of the reverse implication of Theorem 4.6 up to (3) of that proof. Beginning there, let $x_{0} \in M$ and choose $f \in v_{x_{0}}$ and $\varepsilon>0$. Since $v$ is [uniformly] lower semicontinuous in $M$ from the norm topology on $S$ into $S^{\prime}$ with its relativized $p_{N}$ topology, there is a $\delta\left(x_{0}, \varepsilon\right)>0[\delta(\varepsilon)>0]$ such that with $z \in M$ and $\left\|x_{0}-z\right\|<\delta\left(x_{0}, \varepsilon\right),[\delta(\varepsilon)]$, there exists a $g \in v_{z}$ such that $p_{N}(f-g)<\varepsilon$. According to inequality (2) of the proof of Theorem 4.6 there is an $\eta\left(x_{0}, \varepsilon\right)>0[\eta(\varepsilon)>0]$ such that $|t|<\eta\left(x_{0}, \varepsilon\right)$ $[|t|<\eta(\varepsilon)]$ implies that

$$
\left\|\frac{x_{0}+t \alpha y}{\left\|x_{0}+t \alpha y\right\|}\right\|-x_{0} \quad<\delta\left(x_{0}, \varepsilon\right), \quad[\delta(\varepsilon)]
$$

for all $y \in N$ and all $0 \leqq \alpha \leqq 1$ [for all $x_{0} \in M$, all $y \in N$, and all $0 \leqq \alpha \leqq 1$ ]. Now let $t_{0}$ be a fixed real number such that $\left|t_{0}\right|<\eta\left(x_{0}, \varepsilon\right)\left[\left|t_{0}\right|<\eta(\varepsilon)\right]$ and let $y_{0}$ be an arbitrary but then fixed element of $N$. Then except for a set of Lebesgue measure zero which depends on $x_{0}, y_{0}$, and $t_{0}, G\left(\left(x_{0}+t_{0} \alpha y_{0}\right) /\left\|x_{0}+t_{0} \alpha y_{0}\right\|, y_{0}\right)$ exists. The same conclusions can be drawn from this as were drawn in the proof of Theorem 4.6 and so for all $t$ such that $|t|<\eta\left(x_{0}, \varepsilon\right)[|t|<\eta(\varepsilon)]$ 


$$
\left|\frac{\left\|x_{0}+t y_{0}\right\|-\left\|x_{0}\right\|}{t}-G\left(x_{0}, y_{0}\right)\right|<\varepsilon .
$$

Since $\eta\left(x_{0}, \varepsilon\right),[\eta(\varepsilon)]$, is independent of $y_{0} \in N$ [of $y_{0} \in N$ and $\left.x_{0} \in M\right]$, the reverse implication of the present theorem is proved.

The following corollary uniformizes Corollary 4.7.

4.10. Corollary. If $M$ is a subset of $S$ then $\|\cdot\|$ is differentiable [uniformly] in $M$ uniformly with respect to $S$ iff $v$ defined on $S$ is [uniformly] lower semicontinuous in $M$ from the norm topology on $S$ into the norm topology on $S^{\prime}$.

4.11. Corollary. $\|\cdot\|$ is Frechet differentiable at a point $x$ on $S$ iff $v$ defined on $S$ is lower semicontinuous at $x$ from the norm topology on $S$ into the norm topology on $S^{\prime}$.

An assertion equivalent to the forward implication of the preceding corollary was stated but proved in a different way in Smulian [4, p. 549]. The following corollary should be compared with Corollaries 4.5 and 4.8 .

4.12. Corollary. Let $C$ be a convex hypersurface contained in $S$. Then the following are equivalent:

(i) $\|\cdot\|$ is [uniformly] Frechet differentiable in $C$;

(ii) $v$ defined on $C$ is single-valued and [uniformly] continuous from the norm topology on $C$ into the norm topology on $S^{\prime}$;

(iii) $v$ defined on $C$ is [uniformly] lower semicontinuous in $C$ from the norm topology on $C$ into the norm topology on $S^{\prime}$.

The next few theorems will generalize some of the previous theorems to subsets of the boundaries of arbitrary symmetric convex bodies.

Let $\|\cdot\|_{1}$ denote the Minkowski functional of a symmetric convex body and let $\mu$ be the map from $S_{1}$ into $S_{1}^{\prime}$ that assigns to each point $x$ in $S_{1}$ the set of elements $f \in S_{1}^{\prime}$ such that $f^{-1}(r)$ for $r>0$ is a supporting hyperplane of $S_{1}$ with point of contact $x$. It is clear that if $x \in S_{1}$ then

and

$$
v_{x}=\left\{\frac{g}{\|g\|}: g \in \mu_{x}\right\}
$$

$$
\mu_{x}=\left\{\frac{f}{\|f\|_{1}}: f \in v_{x}\right\} .
$$

Thus $v_{x}$ is a singleton set iff $\mu_{x}$ is a singleton set.

4.13. Proposition. Let $M$ be a subset of $S_{1}$ and let $v$ be defined on $S_{1}$. Then $v$ is [uniformly] lower semicontinuous in the set $M$ from the norm topology on $S_{1}$ into the norm topology on $S^{\prime}$ iff $\mu$ is [uniformly] lower semicontinuous in the set $M$ from the norm topology on $S_{1}$ into the norm topology on $S_{1}^{\prime}$. 
Proof. To prove the forward implication, let $x \in M$ and $g \in \mu_{x}$. Then $\left(g+\varepsilon U^{\prime}\right) \cap S_{1}^{\prime}$ is a neighborhood of $g$ in the relativized norm topology on $S_{1}^{\prime}$. If it is supposed that $\alpha\|x\| \leqq\|x\|_{1} \leqq \beta\|x\|$ for all $x$ in $X$ then $\alpha\|f\|_{1} \leqq\|f\| \leqq \beta\|f\|_{1}$. Let $f \in S^{\prime}$ and $g \in S^{\prime}{ }_{1}$. Then

$$
\left\|\frac{f}{\|f\|_{1}}-g\right\| \leqq \frac{2 \beta^{2}}{\alpha}\left\|_{1} f-\frac{g}{\|g\|}\right\| .
$$

Now $g /\|g\| \in v_{x}$ and $\left(g /\|g\|+\left(\alpha \varepsilon / 4 \beta^{2}\right) U^{\prime}\right) \cap S^{\prime}$ is a neighborhood of an element in $v_{x}$ in the norm topology on $S^{\prime}$. Since $v$ is [uniformly] lower semicontinuous at $x$ [in the set $M]$ there is a $\delta(x, \varepsilon)$, $[\delta(\varepsilon)]$, such that $x^{\prime}$ in $S_{1}$ and $\left\|x-x^{\prime}\right\|<\delta(x, \varepsilon),[\delta(\varepsilon)]$ implies that

$$
v_{x^{\prime}} \cap \frac{g}{\|g\|}+\frac{\alpha \varepsilon}{4 \beta^{2}} U^{\prime} \cap S^{\prime} \neq \varnothing .
$$

Thus if $f$ is in this intersection then $f /\|f\|_{1} \in \mu_{x^{\prime}}$ and

$$
\left\|\frac{f}{\|f\|_{1}}-g\right\| \leqq \frac{2 \beta^{2}}{\alpha}\left\|f-\frac{g}{\|g\|}\right\|<\varepsilon .
$$

Hence if $x^{\prime}$ is on $S_{1}$ and $\left\|x-x^{\prime}\right\|<\delta(x, \varepsilon),[\delta(\varepsilon)]$, then $\mu_{x^{\prime}} \cap g+\varepsilon U^{\prime} \cap S_{1}^{\prime} \neq \varnothing$. Thus $\mu$ is [uniformly] lower semicontinuous in the set $M$ in the relevant topologies.

To prove the reverse implication, let $x \in M$ and $f \in v_{x}$. Then $\left(f+\varepsilon U^{\prime}\right) \cap S^{\prime}$ is a neighborhood of an element in $v_{x}$. But also $\left(f /\|f\|_{1}+\left(\alpha^{2} \varepsilon / 4 \beta\right) U^{\prime}\right) \cap S_{1}^{\prime}$ is a neighborhood of an element in $\mu_{x}$ in the norm topology on $S_{1}^{\prime}$. Since $\mu$ is [uniformly] lower semicontinuous at $x$ [in the set $M]$ there is a $\delta(x, \varepsilon),[\delta(\varepsilon)]$, such that with $x^{\prime}$ in $S_{1}$ and $\left\|x-x^{\prime}\right\|<\delta(x, \varepsilon),[\delta(\varepsilon)]$,

$$
\mu_{x^{\prime}} \cap \frac{f}{\|f\|_{1}}+\frac{\alpha^{2} \varepsilon}{4 \beta} U^{\prime} \cap S_{1}^{\prime} \neq \varnothing .
$$

Thus if $g$ is an element in this intersection then $g /\|g\| \in v_{x}$, and

$$
\left\|f-\frac{g}{\|g\|}\right\| \leqq \frac{2 \beta}{\alpha^{2}}\left\|\frac{f}{\|f\|_{1}}-g\right\|<\varepsilon .
$$

Hence $v_{x^{\prime}} \cap f+\varepsilon U^{\prime} \cap S^{\prime} \neq \varnothing$ and $v$ is [uniformly] lower semicontinuous at $x$ [in the set $M$ ] in the relativized norm topologies.

The following theorem generalizes Corollary 4.10 to subsets of the boundary of an arbitrary convex body

4.14. THEOREM. If $M$ is a subset of $S_{1}$ then $\|\cdot\|_{1}$ is differentiable [uniformly] in $M$ uniformly with respect to $S_{1}$ iff $v$ defined on $S_{1}$ is [uniformly] lower semicontinuous in $M$ from the norm topology on $S_{1}$ into the norm topology on $S^{\prime}$. 
Proof. The proof is made by first using Corollary 4.10 and then Proposition 4.13.

The following corollary generalizes Corollary 4.11 .

4.15. COROllary. $\|\cdot\|_{1}$ is Frechet differentiable at a point $x$ on $S_{1}$ iff $v$ defined on $S_{1}$ is lower semicontinuous at $x$ from the norm topology on $S_{1}$ into the norm topology on $S^{\prime}$.

The following theorem generalizes Corollary 4.12 .

4.16. Theorem. Let $C$ be a convex hypersurface contained in $S_{1}$. Then the following are equivalent:

(i) $\|\cdot\|_{1}$ is [uniformly] Frechet differentiable in $C$;

(ii) $v$ defined on $C$ is single-valued and [uniformly] continuous from the norm topology on $C$ into the norm topology on $S^{\prime}$;

(iii) $v$ defined on $C$ is [uniformly] lower semicontinuous in $C$ from the norm topology on $C$ into the norm topology on $S^{\prime}$.

Proof. Corollary 4.5, the remark made just before Proposition 4.13, Theorem 4.14 and the fact that lower semicontinuity and continuity coincide for singlevalued functions shows that (i) implies (ii) and also shows that (ii) implies (iii). That (iii) implies (i) follows from Theorem 4.14.

4.17. Definition. Let $v$ be the spherical image map defined on $S$. Define the extended spherical image map $T$ from $X$ into $X^{*}$ by setting

$$
T x= \begin{cases}\|x\| v_{x /\|x\|}, & x \neq \theta \\ \theta, & x=\theta\end{cases}
$$

for each $x$ in $X$.

It is well known that if $X$ is a real Hilbert space then $T$ is a linear isometry between $X$ and $X^{*}$. Klee [2, p. 35] has proposed the following problem: Characterize intrinsically those spaces $X$ for which $T$ is a homeomorphism of $X$ onto $X^{*}$ in the norm topologies.

4.18. THEOREM. $X$ and $X^{*}$ with their norm topologies are homeomorphic under the extended spherical image map $T$ iff $X$ is a Banach space, $\|\cdot\|$ is Frechet differentiable, and $U$ is weakly uniformly convex.

Proof. To prove the forward implication recall that Klee [1] has proved that a Banach space cannot be homeomorphic with a noncomplete normed linear space. Thus $X$ must be a Banach space. The fact that $T$ is onto means, according to James' criterion, that $X$ is reflexive The continuity of $T$ and $T^{-1}$ then implies by Corollary 4.12 that $\|\cdot\|$ and $\|\cdot\|^{*}$ are Frechet differentiable. By (ii) of Corollary $3.18, S$ is weakly uniformly rotund or $U$ is weakly uniformly convex.

To prove the reverse implication note that by Theorem $5.4 X$ is reflexive so that $T$ is onto. $T$ is single-valued and continuous in the relevant topologies according 
to Corollary 4.12. By (ii) of Corollary $3.18\|\cdot\|^{*}$ is Frechet differentiable and so the same can be said of $T^{-1}$. Thus $T$ is a homeomorphism between $X$ and $X^{*}$ with their norm topologies.

5. Complements and further problems. This section is concerned with ancillary results and comparisons which would have confused the exposition had they been inserted in the main discussion. Connections with the work of previous authors are mentioned and analyzed. A discussion of further questions and problems in the development of the geometry of infinite dimensional spaces closes the paper.

After Smulian [3] showed that uniform convexity was dual to the uniform Frechet differentiability of the norm functional, other investigators sought the appropriate weakening of uniform convexity which would be dual to Frechet differentiability of the norm functional. Lovaglia [1] advanced a localization of uniform convexity called local uniform convexity which proved to be too strong to yield the required duality in the sense that with $U$ weakly compact, the local uniform convexity of $U\left[U^{\prime}\right]$ implies that $\|\cdot\|^{*}[\|\cdot\|]$ is Frechet differentiable, but the converse implication even when $U$ is weakly compact requires an additional hypothesis.

In another attempt to "localize" uniform convexity, Anderson [1] considered the property called midpoint local uniform convexity which had been previously investigated by G. Lumer and by M. M. Day although these last had not been concerned with the duality problem. However midpoint local uniform convexity proved to be too much of a weakening of uniform convexity in the sense that while Frechet differentiability of $\|\cdot\|\left[\|\cdot\|^{*}\right]$ together with the weak compactness of $U$ implies the midpoint local uniform convexity of $U^{\prime}[U]$, Anderson was unable to reverse the implication.

Corollary 3.18 shows that the correct localization of uniform convexity which corresponds to the weakening of the differentiability of the norm functional from uniform Frechet differentiability to Frechet differentiability is the property called weak uniform convexity of the unit ball or weak uniform rotundity of the unit sphere. In this property the localization is in terms of supporting hyperplanes and the depth of the midpoint of the segment is measured "in the direction" of the supporting hyperplane. It is now clear that when $U$ is weakly compact there is complete duality between weak uniform rotundity and Frechet differentiability of the norm functional.

It is interesting to compare weak uniform convexity with the other localization of uniform convexity considered above. Weak uniform convexity will first be compared with local uniform convexity and then with midpoint local uniform convexity.

Let $P$ be the set of all functionals in $X^{*}$ which attain their supremum on $U$. It is shown in Theorem 5.4 below that if $X$ is a Banach space and $U$ is weakly 
uniformly convex, then $P=X^{*}$. On the other hand, since there is a Banach space with a locally uniformly convex unit ball for which $P \neq X^{*}$, cf. Lovaglia [1, Theorem 3.1], it is futile to expect the local uniform convexity of $U$ to imply its weak uniform convexity. However there is one valid implication which is the content of the next theorem. Recall that Bishop and Phelps [1] have shown that if $X$ is a Banach space then $P$ is norm dense in $X^{*}$.

5.1. THEOREM. Let $P$ be the set of all functionals in $X^{*}$ which attain their supremum on $U$. If $U$ is locally uniformly convex then $U$ is weakly uniformly convex at $P$.

Proof. Let $f \in P \cap S^{\prime}$. Then there is an $x_{0}$ in $S$ such that $f\left(x_{0}\right)=1$. Since $U$ is locally uniformly convex, to each $\varepsilon>0$ there corresponds $\delta\left(\varepsilon, x_{0}\right)>0$ such that with $x$ in $S$,

$$
1-\left\|\frac{x_{0}+x}{2}\right\|<\delta\left(\varepsilon, x_{0}\right)
$$

implies $\left\|x_{0}-x\right\|<\varepsilon$. Let $\delta(\varepsilon, f)=\delta\left(\varepsilon / 2, x_{0}\right)$. Then with $x$ and $y$ in $S$,

$$
1-\left|f\left(\frac{x+y}{2}\right)\right|<\delta(\varepsilon, f)
$$

implies $\|x-y\| \leqq\left\|x_{0}-x\right\|+\left\|x_{0}-y\right\|<\varepsilon / 2+\varepsilon / 2=\varepsilon$. Since $f$ was an arbitrary element of $P \cap S^{\prime}$, this shows that $U$ is weakly uniformly convex at $P$.

5.2. CoRollary. Suppose that $U$ is both weakly compact and locally uniformly convex. Then $U$ is weakly uniformly convex.

5.3. THEOREM. If $U$ is weakly uniformly convex then $U$ is midpoint locally uniformly convex.

Proof. Let $x_{0}$ be in $S$ and let $f_{0}$ be any element in $S^{\prime}$ such that $f_{0}\left(x_{0}\right)=1$. Suppose $\varepsilon>0$ is given. Then using the weak uniform convexity of $U$, there is a $\delta\left(\varepsilon, f_{0}\right)>0$ such that

$$
1-\left|f_{0}\left(\frac{x+y}{2}\right)\right|<\delta\left(\varepsilon, f_{0}\right)
$$

implies $\|x-y\|<\varepsilon$ where $x$ and $y$ are in $U$. Now if $x$ and $y$ are in $U$ then

$$
\left\|x_{0}-\frac{x+y}{2}\right\|<\delta\left(\varepsilon, f_{0}\right)
$$

implies

$$
\begin{aligned}
1-\left|f_{0}\left(\frac{x+y}{2}\right)\right| & \leqq\left|f_{0}\left(x_{0}-\frac{x+y}{2}\right)\right| \\
& \leqq\left\|x_{0}-\frac{x+y}{2}\right\|<\delta\left(\varepsilon, f_{0}\right)
\end{aligned}
$$


so that $\|x-y\|<\varepsilon$. Setting $\delta\left(\varepsilon, x_{0}\right)=\delta\left(\varepsilon, f_{0}\right)$ it is clear that $U$ is midpoint locally uniformly convex.

In a different vein recall that Smulian [1] has investigated a sequentialized implication of uniform convexity which is called 2-rotundity here. Subsequently, Fan and Glicksberg [1] considered a generalization of 2-rotundity which is called full $k$-convexity in that paper but which is called $k$-rotundity here. In order to understand the implications for weak compactness of the three localizations and directionalizations of uniform convexity it will be helpful to consider the localization and directionalization of $k$-rotundity. The arrows in the following diagram are implications and are to be interpreted in the following sense $: I \rightarrow J$ means that if the unit sphere in a normed space has property $I$, then it has property $J$. Note that the property $k$ RD, i.e., $k$-rotundity in any direction is called weak full $k$-convexity in Fan and Glicksberg [1].

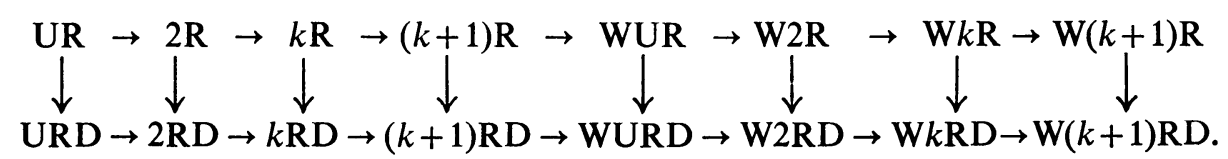

The reader will have no trouble proving these implications.

The following theorem sheds some light on the weak compactness of a unit ball which satisfies one of the localizations or directionalizations of uniform convexity or $k$-rotundity.

5.4. THEOREM. In the normed linear space $X$ :

(i) if $S$ is weakly k-rotund, then $U$ is weakly compact iff $X$ is norm complete;

(ii) if $S$ is weakly $k$-rotund in each direction, then $U$ is weakly compact iff $X$ is weakly sequentially complete.

Proof. The proofs of both assertions will be carried along simultaneously with brackets to indicate that (ii) is being discussed.The forward implications are well known so that only the reverse implications will be proved here and this will be done using James' criterion for the weak compactness of the unit ball of a Banach space. Let $f$ be an element in $S^{\prime}$ and suppose $\left\{x_{n}\right\}$ is a sequence on $S$ such that $\lim _{n} f\left(x_{n}\right)=1$. Then

$$
\lim _{n_{1}, \cdots, n_{k} \rightarrow \infty}\left|\frac{1}{k} f\left(x_{n_{1}}+\cdots+x_{n_{k}}\right)\right|=1
$$

so that since $S$ is weakly $k$-rotund [weakly $k$-rotund in each direction] at $f$, the sequence $\left\{x_{n}\right\}$ is a Cauchy sequence in norm [weak Cauchy sequence]. Since by hypothesis the space is norm complete [weakly sequentially complete], there is an $x_{0}$ in $X$ such that $\lim _{n} x_{n}=x_{0}$ in the norm [weak] topology. Thus $f\left(x_{0}\right)=\lim _{n} f\left(x_{n}\right)=1$ so that $x_{0}$ is a point of contact of the closed supporting hyperplane $f^{-1}(1)$ with $S$. Since $f$ was an arbitrary element on $S^{\prime}$ it follows that 
every continuous linear functional attains its supremum on $U$. In both cases James' criterion can be applied to give the weak compactness of $U$.

From Theorem 5.4 it follows that the separable space $l_{1}$ does not have a symmetric convex body which is weakly $k$-rotund in each direction. Thus a separable normed linear space need not have symmetric convex bodies which satisfy any of the localizations or directionalizations of uniform rotundity or $k$-rotundity.

Before leaving weak uniform convexity the connection between this property and weak uniform convexity in each direction will be illuminated.

5.5. TheоRem. Let $X$ be a Banach space. Then $S$ is weakly uniformly rotund iff $S$ is weakly uniformly rotund in each direction, $S$ has property (A), and $U$ is weakly compact.

Proof. To prove the forward implication let $\left\{x_{n}\right\}$ be a sequence on $S$ converging weakly to $\left\{x_{0}\right\}$ on $S$. Let $f_{0}$ be in $S$ such that $f_{0}\left(x_{0}\right)=1$. Then $\lim _{n} f\left(x_{n}\right)=1$. Define a new sequence $\left\{y_{n}\right\}$ on $S$ by setting

$$
y_{n}= \begin{cases}x_{(n+1) / 2}, & n \text { odd }, \\ x_{0}, & n \text { even } .\end{cases}
$$

Then $\lim _{n} f\left(y_{n}\right)=1$ so that by (i) of Theorem $3.20,\left\{y_{n}\right\}$ is a Cauchy sequence. Hence $\left\{x_{n}\right\}$ converges in norm to $x_{0}$ and $S$ has property (A).

To prove the reverse implication let $\left\{x_{n}\right\}$ be a sequence in $S$ for which there is an $f_{0}$ in $S^{\prime}$ such that $\lim _{n} f_{0}\left(x_{n}\right)=1$. By (i) of Theorem 3.20, because $S$ is weakly uniformly rotund in each direction, the sequence $\left\{x_{n}\right\}$ is a weak Cauchy sequence. Since $X$ is reflexive it is weakly sequentially complete and so there is an $x_{0}$ which is the weak limit of $\left\{x_{n}\right\}$. Now $\left\|x_{0}\right\| \leqq \lim _{n} \inf \left\|x_{n}\right\|=1$, butalso $f_{0}\left(x_{0}\right)$ $=\lim _{n} f_{0}\left(x_{n}\right)=1$ so that $\left\|x_{0}\right\|=1$. Since $S$ has property (A), $\left\{x_{n}\right\}$ converges in norm to $x_{0}$ and hence $\left\{x_{n}\right\}$ is a Cauchy sequence in norm. By (i) of Theorem 3.20, $S$ is weakly uniformly rotund at $f_{0}$. Since $f_{0}$ was an arbitrary element in $S^{\prime}$ it follows that $S$ is weakly uniformly rotund.

The various connections between rotundity and weak uniform rotundity in a direction will now be examined.

5.6. Proposition. Let $K$ be a closed linear subspace of $X$ of finite deficiency. Then the unit sphere $S$ of $X / K$ is rotund iff the unit sphere $S$ of $X$ is weakly uniformly rotund at $K^{\perp}$ in the directions $K^{\perp}$.

Proof. To prove the forward implication let $\delta(\varepsilon, K)$ be the modulus of rotundity given by the hypothesis and let $f$ and $g$ be in $K^{\perp} \sim\{\theta\}$. Then if $x$ and $y$ are in $S$, then $\bar{x}$ and $\bar{y}$ are in $\bar{U}$ so

implies

$$
\left\|\frac{\bar{x}+\bar{y}}{2}\right\|=\left\|\overline{\frac{x+y}{2}}\right\| \geqq\left|\frac{f}{\|f\|}\left(\frac{x+y}{2}\right)\right|>1-\delta(\varepsilon, K)
$$




$$
\left|\frac{g}{\|g\|}(x-y)\right| \leqq\|\bar{x}-\bar{y}\|<\varepsilon .
$$

Set $\delta(\varepsilon, f, g)=\|f\| \delta\left(\|g\|^{-1} \varepsilon, K\right)$. Then with $x$ and $y$ in $S,|g(x-y)| \geqq \varepsilon$ implies $\|f\|-1 / 2|f(x+y)| \geqq \delta(\varepsilon, f, g)$.

Conversely, suppose $S$ is not rotund. Then there are points $\bar{x}$ and $\bar{y}$ on $\bar{S}$ such that $\|\bar{x}+\bar{y}\|=2$ and $\bar{x} \neq \bar{y}$. Then there are $f$ and $g$ in $K^{\perp} \cap S^{\prime}$ such that with $u$ in $\bar{x}$ and $v$ in $\bar{y}, f(u+v)=2$ and $g(u-v)>2 \varepsilon>0$. Let $0<\delta<1$ and choose $u$ in $\bar{x}$ and $v$ in $\bar{y}$ such that $1+\delta>\|v\| \geqq\|u\| \geqq 1$. Then

but

$$
1-\frac{1}{2}\left|f\left(\frac{u}{\|u\|}+\frac{v}{\|v\|}\right)\right| \leqq 1-\frac{1}{\|v\|} \leqq\|v\|-1<\delta
$$

$$
\left|g\left(\frac{u}{\|u\|}-\frac{v}{\|v\|}\right)\right| \geqq\|v\|^{-1} g(u-v)>\|v\|^{-1} 2 \varepsilon>\varepsilon .
$$

Since $u /\|u\|$ and $v /\|v\|$ are in $S$, and $\delta$ can be made arbitrarily small, it follows that $S$ is not weakly uniformly rotund at $f$ in the direction $g$. Since $f$ and $g$ are in $K^{\perp}, S$ is not weakly uniformly rotund at $K^{\perp}$ in the directions $K^{\perp}$. This completes the proof of the proposition.

Note that if there is a common modulus of rotundity for all the two dimensional quotient spaces of $X$ then the first part of the proof of Proposition 5.6 can be used to conclude that $X$ is uniformly convex. This was first shown in a different way in Day $[1$, p. 382].

The next theorem gives the connection between weak uniform rotundity in a direction and the rotundity of unit spheres of quotient spaces.

5.7. THEOREM. (i) $S$ is weakly uniformly rotund in each direction iff every two dimensional quotient space of $X$ with a weakly closed zero element has a rotund unit sphere. (ii) $S^{\prime}$ is weakly uniformly rotund at $Q(X)$ in the directions $Q(X)$ iff every two dimensional quotient space of $X^{*}$ with a weak* closed zero element has a rotund unit sphere.

Proof. The proofs of both assertions will be carried out simultaneously with brackets to indicate that assertion (ii) is being discussed. Let $f$ and $g$ [ $Q x$ and $Q y]$ be linearly independent elements in $X^{*}[Q(X)]$. Then with $K=f^{-1}(0) \cap g^{-1}(0)$ $\left[L=(Q x)^{-1}(0) \cap(Q y)^{-1}(0)\right]$, it is clear $K^{\perp}\left[L^{\perp}\right]$ is the linear hull of $f$ and $g$ $[Q x$ and $Q y]$. Then $X / K\left[X^{*} / L\right]$ is a quotient space of the type described in assertion (i) [(ii)]. Furthermore every such quotient space of $X\left[X^{*}\right]$ can be so obtained. Since $f$ and $g$ [Qx and $Q y]$ were linearly independent but otherwise arbitrary elements of $X^{*}[Q(X)]$, the theorem follows from Proposition 5.6.

The remainder of the theorems in this section will be concerned with the implications between weak uniform rotundity in directions and the rotundity of the surface of the unit sphere itself. 
5.8. THEOREM. $S^{\prime}$ is weakly uniformly rotund at $Q(X)$ in the directions $Q(X)$ iff $S^{\prime}$ is $Q(X)$-rotund.

Proof. To prove the forward implication suppose that $S^{\prime}$ is not $Q(X)$-rotund. Then there are points $f$ and $g$ in $S^{\prime}$ and $Q x$ and $Q y$ in $S^{\prime \prime}$ such that $Q x(f+g)=2$ but $|Q y(f-g)|>0$. Then $S^{\prime}$ is not weakly uniformly rotund at $Q x$ in the direction $Q y$.

To show the reverse implication suppose that $S^{\prime}$ is not weakly uniformly rotund at $Q(X)$ in the directions $Q(X)$. Then there are elements $Q x$ and $Q y$ in $S^{\prime \prime}, \varepsilon>0$, and sequences $\left\{f_{n}\right\}$ and $\left\{g_{n}\right\}$ in $S^{\prime}$ such that $\left|Q y\left(f_{n}-g_{n}\right)\right| \geqq \varepsilon$ but $2-Q x\left(f_{n}+g_{n}\right)<2 n^{-1}$ for all $n$. Let $f_{0}$ and $g_{0}$ be weak* cluster points of $\left\{f_{n}\right\}$ and $\left\{g_{n}\right\}$ respectively. Since $Q x$ is weak* continuous, $1 / 2\left(f_{0}+g_{0}\right)$ is a $Q(X)$ support point on $S^{\prime}$ such that the $Q(X)$-hyperplane, $(Q x)^{-1}(1)$, supporting $S^{\prime}$ at $1 / 2\left(f_{0}+g_{0}\right)$ intersects $U^{\prime}$ at the distinct points $f_{0}$ and $g_{0}$ of $S^{\prime}$. Thus $1 / 2\left(f_{0}+g_{0}\right)$ is not a $Q(X)$-rotund point and therefore $S^{\prime}$ is not $Q(X)$-rotund.

5.9. THEOREM. (i) If $S$ is weakly uniformly rotund in each direction then $S$ is rotund. The converse is not true even in a conjugate space. (ii) If $S^{\prime}$ is rotund then $S^{\prime}$ is weakly uniformly rotund at $Q(X)$ in the directions $Q(X)$. The converse is not true.

The counter-examples for Theorem 5.9 are the same as those given after the proof of Theorem 2.4. Note that the first counter-example discussed there is in $l_{1}$ which is congruent to a conjugate space thus providing the counter-example for (i). The counter-example for (ii) is provided by the second counter-example discussed in $\$ 2$ and by the equivalence between weak uniform rotundity at $Q(X)$ in each of the directions $Q(X)$, and $Q(X)$-rotundity established in Theorem 5.8. Notice also that this counter-example shows that there is a conjugate space with a symmetric convex body whose induced norm makes all two dimensional quotient spaces with a weak* closed zero element strictly convex but does not make all two dimensional quotient spaces with a weakly closed zero element strictly convex.

Among the questions raised by this paper at least two seem worthy of mention. The first concerns geometric properties defined by seminorms in arbitrary locally convex spaces. The absolute values of continuous linear functionals are the seminorms for the weak topology on a normed linear space. These particular seminorms were used to define geometric properties in the normed space. Now one of the uses of geometric properties in the past has been in the classification of normed linear spaces. How generally is this possible for locally convex spaces? More specifically, can the localizations and directionalizations of uniform convexity be used somehow to single out important classes of locally convex spaces or is the theory simply inapplicable to arbitrary locally convex spaces? Note that in the event of applicability not only would arbitrary locally convex spaces be classified, but also along with them arbitrary normed spaces since some of the 
geometric properties considered in this paper prove to be equivalent formulations of other geometric properties which have long been used to classify Banach spaces.

The second problem concerns curvature and convexity. One of the fruitful results of the classical differential geometry of manifolds modeled on finite dimensional spaces is the theory of integral curvature as outlined for example in Busemann $[1$, p. 26]. Can a corresponding theory of integral curvature for infinite dimensional manifolds be developed in spite of the well-known difficulties involved in defining rotationally invariant countably additive measures in infinite dimensional Hilbert space? One thing appears certain however and that is the existence of a theory of curvature not involving integrals and measures but only distances measured by seminorms. In particular, each of the localization and directionalizations of uniform convexity, generates a new modulus of convexity. It ought to be possible to find dual moduli of smoothness in analogy to the modulus of uniform smoothness defined and shown by Day [1] to be dual to the modulus of uniform convexity.

K. W. ANDERSON

\section{REFERENCES}

1. Midpoint local uniform convexity, and other geometric properties of Banach spaces Doctoral dissertation, Univ. of Illinois, Urbana, Illinois, 1960, $48 \mathrm{pp}$.

G. AsColi

1. Sugli spazi lineari metrici e le loro varietà lineari, Ann. Mat. Pura Appl. (4) 10 (1932), 33-81. E. Bishop AND R. R. PHel PS

1. A proof that every Banach space is subreflexive, Bull. Amer. Math. Soc. 67 (1961), 97-98, H. F. BOHNENBLUST AND S. KARLIN

1. Geometrical properties of the unit sphere in Banach algebras, Ann. of Math. (2) 62 (1955). 217-229.

H. BusemanN

1. Convex surfaces, Interscience, New York, 1958.

J. A. Clarkson

1. Uniformly convex spaces, Trans. Amer. Math. Soc. 40 (1936), 396-414.

D. F. CUDIA

1. Rotundity, Proc. Sympos. Pure Math., Vol. 7, Amer. Math. Soc., Providence, R.I., 1963.

M. M. DAY

1. Uniform convexity in factor and conjugate spaces, Ann. of Math. (2) 45 (1944), 375-385.

2. Strict convexity and smoothness of normed spaces, Trans. Amer. Math. Soc. 78 (1955), 516-528.

3. Normed linear spaces, Springer, Berlin, 1958.

K. FAN AND I. GLicksberg

1. Fully convex normed linear spaces, Proc. Nat. Acad. Sci. U.S.A. 41 (1955), 947-953.

2. Some geometrical properties of the spheres in a normed linear space, Duke Math. J. 25 (1958), 553-568.

R. C. JAMES

1. Reflexivity and the supremum of linear functionals, Ann. of Math. (2) 66 (1957), 159-169.

2. Characterizations of reflexivity, Studia Math. (to appear). 


\section{L. KLEE}

1. Invariant metrics in groups (solution of a problem of Banach), Proc. Amer. Math. Soc. 3 (1952), 484-487.

2. Convex bodies and periodic homeomorphisms in Hilbert space, Trans. Amer. Math. Soc. 74 (1953), 10-43.

3. Some new results on smoothness and rotundity in normed linear spaces, Math. Ann. 139 (1959), 51-63.

M. A. Krasnosel'skí and Ya. B. RutickiI

1. Convex functions and Orlicz spaces, Noordhoff, Gröningen, 1961. (English transl. of first Russian edition, 1958).

A. R. Lovaglia

1. Locally uniformly convex Banach spaces, Trans. Amer. Math. Soc. 78 (1955), 225-238. S. MAzUR

1. Uber konvexe Mengen in linearen normierten Räumen, Studia Math. 4 (1933), 70-84.

A. F. Ruston

1. A note on convexity in Banach spaces, Proc. Cambridge Philos. Soc. 45 (1949), 157-159. V. L. Šmulian

1. On some geometrical properties of the sphere in a space of the type (B), Dokl. Akad. Nauk SSSR 24 (1939), 648-652.

2. On some geometrical properties of the unit sphere in the space of the type (B), Mat. Sb. (N. S.) 6 (48) (1939), 77-94. (Russian).

3. Sur la dérivabilité de la norme dans l'espace de Banach, Dokl. Akad. Nauk SSSR 27 (1940), 643-648.

4. Sur la structure de la sphère unitaire dans l'espace de Banach, Mat. Sb. (N. S.) 9 (51) (1941), 545-561.

\section{UNIVERSTTY OF ILLINOIS,}

UrbaNA, IlLINOIS 